\section{\$25. Stimulated Electron-Acoustic-Wave Scattering in a Laser Plasma}

Nikolić, Lj. (The Grad. Univ. for Advanced Studies), Ishiguro, S., Škorić, M.M. (Vinča Inst., Yugoslavia), Takamaru, H., Sato, T. (Earth Simulator Center)

The propagation of a laser light through an underdense plasma is an active research topic. In order to explain high reflectivities observed in experiments, we investigate excitation and growth of electron-acoustic waves. From our simulation data stimulated electronacoustic-wave scattering (SEAS) is identified as a resonant three-wave parametric interaction [1] involving the laser pump $\left(\omega_{0}, k_{0}\right)$, the backscattered lightwave $\left(\omega_{s}, k_{s}\right)$ and the trapped electron-acoustic wave (EAW) $\left(\omega_{a}, k_{a}\right)$. In the linear instability stage, resonant conditions $\omega_{0}=\omega_{s}+\omega_{a}$ and $k_{0}=-k_{s}+k_{a}$ are well satisfied, while electromagnetic waves (pump and Stokes wave) satisfy standard dispersion equation $\omega_{0, s}^{2}=\omega_{p}^{2}+c^{2} k_{0, s}^{2}$. The backscattered wave is always found to be driven near critical, i.e. $\omega_{s} \approx \omega_{p}$ which implies $k_{s} \approx 0$ and $V_{s} \approx 0\left(\omega_{p}\right.$ is the plasma frequency and $V_{s}=c^{2} k_{s} / \omega_{s}$ is the light group velocity). The above decay scheme is observed for a wide range of laser intensities, plasma densities and temperatures. It is known that the high temperatures can significantly change the growth rates and sometimes suppress parametric instabilities. However, according to [2], efficient excitation of trapped EAW $\left(\omega_{a}<\omega_{p}\right)$, is expected in the range $v_{p h} / v_{t}=1-2$ $\left(v_{p h}\right.$ and $v_{t}=(T / m)^{1 / 2}$ are the phase and electron thermal velocities). Thus, for SEAS excitation at the threshold, high thermal velocities which closely match the EAW phase velocity are important.

In Fig. $1 \mathrm{EM}$ and ES spectra of fully developed SEAS are shown. The density and the plasma length are $n=0.9 n_{c r}\left(n_{c r}=n\left(\omega_{0} / \omega_{p}\right)^{2}\right)$ and $L=50 c / \omega_{0}$, respectively, the temperature is $T=500 \mathrm{eV}$ and the laser strength is $\beta=\left(e E_{0}\right) /\left(m c \omega_{0}\right)=0.4\left(E_{0}\right.$ is the amplitude of the electric field). The backscattered EM wave grows at the electron plasma frequency $\omega_{p} \approx 0.85 \omega_{0}$, while corresponding EAW is at $\omega_{0}-\omega_{p} \approx 0.15 \omega_{0}$. Note that apart from ES noise around a natural plasma mode $\left(\omega_{p} \approx 0.85 \omega_{0}\right)$, ponderomotively driven nonresonant modes are also present at 2-nd, $\omega=2 \omega_{0}$ and $k=2 k_{0}$, as well as at zero-harmonic.

The temperature effect can be clearly seen near the threshold intensity for SEAS $(\beta \sim 0.3)$. Since the longitudinal thermal velocity of electrons can easily increase due to e.g. the Raman instability, the temperature in transverse direction was set to $500 \mathrm{eV}$, with a longitudinal temperature taken as a control parameter. Just above the threshold, high electron temperature may be essential for an instability growth. This is illustrated by Fig. 2 in which reflectivities are shown for $\beta=0.3, n=0.4 n_{c r}, L=40 c / \omega_{0}$ at several temperatures, $v_{t} / c=0.19,0.20,0.28$ and 0.30 . There is an optimum temperature for perfect matching with an excited EAW which results in a maximum SEAS reflectivity. For $v_{t} / c=0.2$ observed reflectivity is very high nearly $140 \%$ of the incident laser light. One calculates $v_{p h} / v_{t} \approx 2.64,2.50,1.84$ and 1.72 for $v_{t} / c=0.19$, $0.20,0.28$ and 0.30 , respectively. For temperatures $\leq v_{t} / c=0.18$ and $\beta=0.3$ the instability was not observed during time period of $t \omega_{0}=5000$.
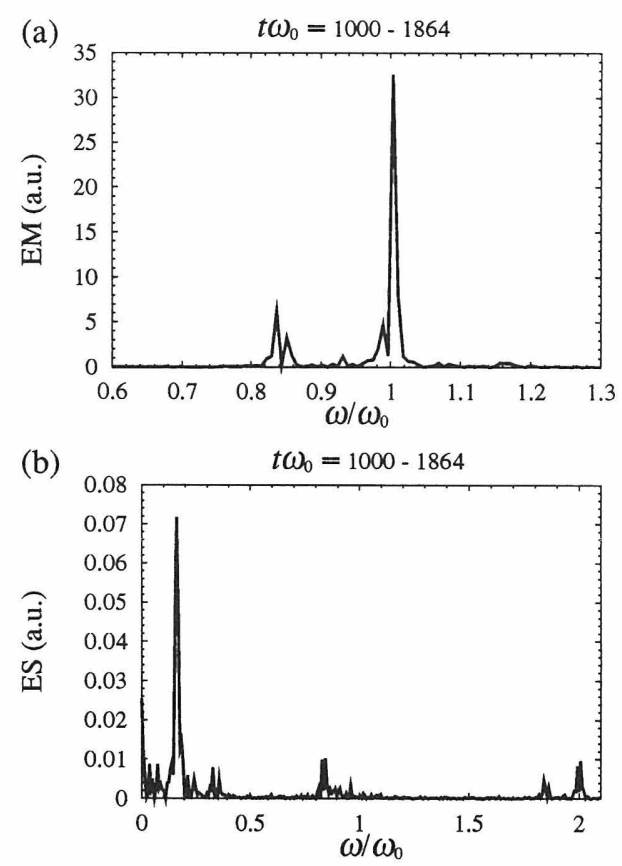

Fig. 1. Spectrum of electromagnetic (a) and electrostatic (b) waves in the plasma layer for time interval $t \omega_{0}=1000-1864$.

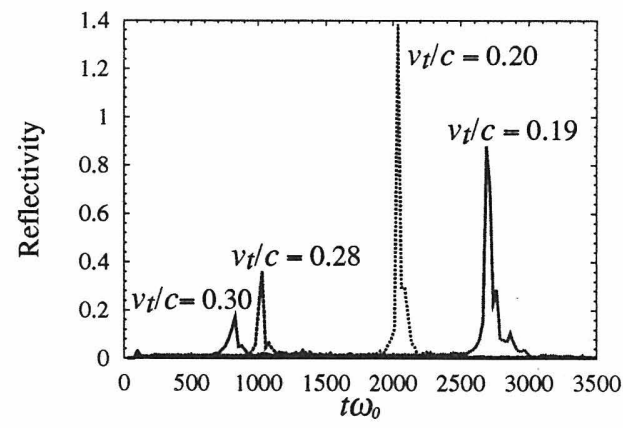

Fig. 2 Reflectivity in time from the plasma layer for different initial electron thermal velocities $v_{t} / c$.

\section{References}

1) K. Mima and K. Nishikawa, in Basic Plasma Physics II, edited by A. A. Galeev and R. N. Sudan, (NorthHolland, Amsterdam 1984), p. 451.

2) H. A. Rose and D. A. Russell, Phys. Plasmas 8, 4784 (2001). 